\title{
24.77 Pflops on a Gravitational Tree-Code to Simulate the Milky Way Galaxy with 18600 GPUs
}

\author{
Jeroen Bédorf \\ Leiden Observatory \\ Email: bedorf@strw.leidenuniv.nl
}

Keigo Nitadori
RIKEN AICS
Email: keigo@riken.jp

\author{
Evghenii Gaburov \\ SURFsara Amsterdam
}

Email: evghenii.gaburov@surfsara.nl

Tomoaki Ishiyama

CCS, University of Tsukuba

Email: ishiyama@ccs.tsukuba.ac.jp

\author{
Michiko S. Fujii \\ National Astronomical Observatory of Japan \\ Email: michiko.fujii@nao.ac.jp
}

Simon Portegies Zwart

Leiden Observatory

Email: spz@strw.leidenuniv.nl

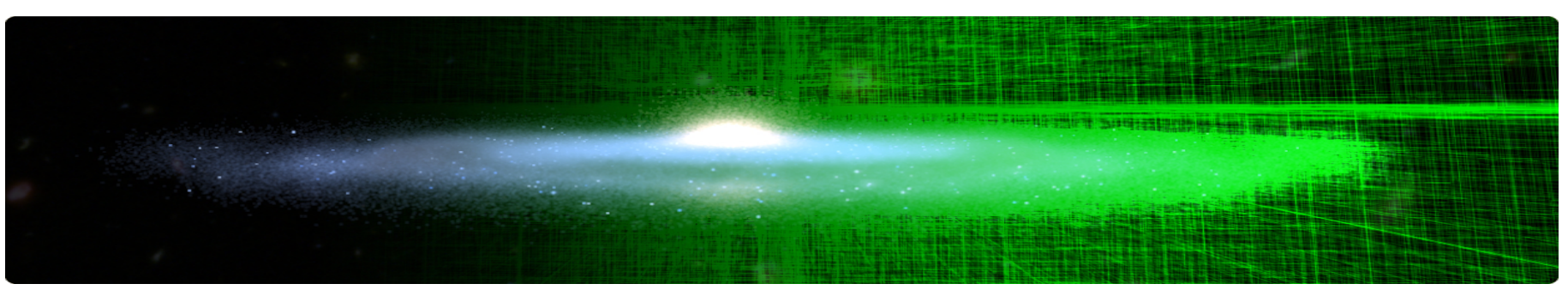

Abstract-We have simulated, for the first time, the long term evolution of the Milky Way Galaxy using 51 billion particles on the Swiss Piz Daint supercomputer with our $N$-body gravitational tree-code Bonsai. Herein, we describe the scientific motivation and numerical algorithms. The Milky Way model was simulated for 6 billion years, during which the bar structure and spiral arms were fully formed. This improves upon previous simulations by using 1000 times more particles, and provides a wealth of new data that can be directly compared with observations. We also report the scalability on both the Swiss Piz Daint and the US ORNL Titan. On Piz Daint the parallel efficiency of Bonsai was above $95 \%$. The highest performance was achieved with a 242 billion particle Milky Way model using 18600 GPUs on Titan, thereby reaching a sustained GPU and application performance of 33.49 Pflops and 24.77 Pflops respectively.

Submitted in the categories: Scalability, Time-to-solution and Peak performance

\section{INTRODUCTION}

The Sun, although the bright centre of the Solar System, is only a tiny speck among the billions of stars that form the disk of our home galaxy, the Milky Way. And, although bright and clearly visible on the night sky, the stars, interstellar gas and planets contain only a minor fraction of the mass of the Galaxy as most of its mass ( $\gtrsim 90 \%$ ) is dark matter. The stellar distribution of the Galaxy is not smooth; the disk is warped, shows spiral patterns, it is thicker in some places, and it is clumpy in others, whereas the central portion of the Milky Way contains a bar-shaped structure composed of stars. The origin of these structures is not known. They may be the remnants from the formation of the Galaxy in its cosmological setting, or such structures may appear spontaneously, possibly due to the interaction between gas and stars in the star-formation cycle,

SC14, November 16-21, 2014, New Orleans

978-1-4799-5500-8/14/\$31.00 (C) 2014 IEEE self-gravity, gravitational relaxation processes or nonlinearities in the finite point-mass dynamics of the Galaxy.

We currently lack the theoretical understanding to fully appreciate the fine-structure dynamics of the Galaxy, and this gap between theory and observations is only about to widen. On 19 December 2013 the European Space Agency launched the Gaia satellite. In 2017, this $€ 1.5$ billion spacecraft will deliver its first catalogue of about 1 billion stars throughout the Galaxy providing measurements of their distances (up to $\sim 28,000$ light-year), space velocities and stellar type. This data will provide us with the properties of the small scale structure of the Milky Way disk. By comparing these observations with simulation models, we can recover the global structure of the Milky Way disk, including the pattern speed and resonances of both the bar and spiral arms. However, it requires selfconsistent simulations of the Galactic disk with at least an order of magnitude more stars than the number of Gaia samples in order to make a reliable comparison. Such high resolution simulations capture the small scale disk structures, whereas in lower resolution simulations the limited number of stars washes out these details of the Galactic disk. This makes it unclear how structures found in these simulations relate to the data that will be gathered by the Gaia satellite. The ultimate goal is to simulate the Galaxy on a star-by-star basis, with 100 billion stars. This one-to-one correspondence between the stars in the Galaxy and the simulation will allow direct comparison with the Gaia catalogue.

The largest $N$-body simulations of the Galaxy today contain about 100 million objects [1]-[4]. These simulations can be categorized in two types, 1) an analytic, static potential dark matter halo and a live ( $N$-body) disk [1], [2] or 2) both a live dark matter halo and a live disk [3], [4]. The former type of simulation allows us to accurately resolve the disk, but it does not capture the disk-to-halo interaction. It is however known 
that angular momentum transfer from disk to halo plays an important role in the formation and evolution of the bar [2]. For the latter simulations it requires at least five times more dark matter than disk particles to accurately model the dark matter halo and its interaction with the disk. As a result we have to increase the total number of particles in the simulations by almost three orders of magnitude, compared to previous work [2], [5], in order to reach the desired resolution of the Galactic disk.

In this paper we present the first results of a simulation of the Milky Way Galaxy with 51 billion particles. It is only for lack of computing time that we have not simulated the Galaxy with 242 billion particles, but we do present timing results and feasibility analysis.

\section{A. Simulating the Galaxy on a star-by-star basis}

By the nature of the gravitational force each star in the Galaxy attracts all the other 100 billion stars. Numerically such gravitational interactions are most accurately simulated via direct $N$-body force evaluations, in which each of the $N$ objects exerts $N-1$ interactions per time-step [6]. With the $\mathcal{O}\left(N^{2}\right)$ force complexity of this algorithm, the calculation of the forces between all stars requires $\sim 10^{23}$ floating point operations. Calculating forces is by far the most expensive operation in the Galaxy simulation; even adding the nuclear evolution of all the stars only adds $\sim 10^{13}$ operations and moving the stars requires $\sim 10^{12}$ operations [7].

To reduce the computational cost of calculating the forces between all the stars, we adopt the hierarchical Barnes-Hut tree algorithm [8]. In this algorithm the distribution of particles is recursively partitioned into octants until the number of particles in an octant is smaller than a critical value (we use 16 [9]). Once a tree-structure is built and its multipole moments are computed, the code proceeds with the force calculation step. For this, we adopt a multipole acceptance criterion parameterized by an opening angle $\theta$ [9] whose purpose is to decide whether or not the substructure in distant octants can be used as a whole. If the opening angle is infinitesimal the tree-code reduces to a rather inefficient direct $N$-body code, otherwise the asymptotic complexity of the gravitational force calculation reduces to $\mathcal{O}(N \log N)$. With such a tree algorithm, it costs $\sim 10^{16}$ floating point operations to calculate all inter-particle forces, although some additional overhead is introduced by building and traversing the tree-structure.

Our choice of the Barnes-Hut tree algorithm, over a Tree Particle Mesh method (TreePM) [10], [11], to simulate the Milky Way Galaxy is motivated by two reasons. First, the TreePM algorithm assumes periodic boundary conditions, which makes it computationally efficient for cosmological simulations. However, to simulate the Milky Way Galaxy we require open boundary conditions which are computationally expensive to use in a TreePM method, thereby defeating its main speed advantage over the Barnes-Hut tree algorithm. Second, the relative accuracy requirement for the gravitational force approximations, imposed by the long-term simulation of the Milky Way, would require a disproportionally large number of grid cells in a TreePM method, which, due to insufficient interconnect bandwidth, would likely cause the parallel FFT operation to become a bottleneck.
The calculation of gravitational forces is by definition an all-to-all operation, and the tree algorithm does not lift the requirement of the global communication in the gravitational $N$-body problem. Even though the tree algorithm considerably reduces the number of interactions, it still requires all processes to communicate with each other, therefore making this a nontrivial task to run such a code efficiently on a distributed memory architecture with many layers of communication that have low-bandwidth and long-latency relative to the raw performance of the compute node.

Here we describe how we solved the communication bottlenecks and how we parallelized the global data-aware tree algorithm to make it suitable for a large GPU-equipped supercomputer. Our ultimate aim is to simulate the Galaxy on a starby-star basis. In this work we report on our simulation of the Milky Way galaxy with an unprecedented high resolution of 51 billion particles on the Piz Daint supercomputer. We further demonstrate that our ultimate aim, subject to computing time availability, can be achieved on the Titan supercomputer and will take about a week to complete. We used two computers for our research, Piz Daint at ETH Switzerland and Titan at ORNL US. The time for these simulations was offered to us by Director's Discretion.

We used 4096 GPU-equipped nodes of Piz Daint to simulate the Milky Way Galaxy for 6 billion years with 51 billion particles. This high accuracy simulation improves upon current state-of-the-art simulations by over 1000 times in particle numbers and over 10 times in spatial resolution, allowing us for the first time to resolve scales that are less than the average distance between the stars in the Solar Neighborhood.

We also conducted an application scalability study on both Piz Daint and Titan using up to 18600 nodes and up to a 242 billion particle Milky Way model. At the pinnacle, we achieved a sustained GPU and application single precision performance of 33.49 Pflops and 24.77 Pflops respectively. (see $\S \mathrm{VI}$ ). Each full step with 242 billion particles takes about 5 seconds of wall-clock time to compute (see Tab./II), and with a time step of 75,000 year, it would take about one week to simulate the entire Milky Way Galaxy on a star-by-star basis for at least 8 billion years (8 Gyr).

\section{QUANTITATIVE DISCUSSION OF CURRENT STATE OF THE ART}

Tree-codes have been used for previous simulations of galaxies with five hundred thousand [12] to a hundred million [3], [13], [14] particles. Each particle in these earlier simulations represents $\gtrsim 2000$ Solar masses $\left(M_{\odot}\right)$. These studies are used to understand the structure of the bar [2], [5], [15], formation and dynamics of spiral arms [3], [16], [17], pitch angle and galactic shear [17], [18], and the warping of the stellar disk [2]. In another recent work the properties of the Milky Way bulge are investigated using simulations with up to 30 million particles [5]. The largest simulations of disk galaxies with a live halo so far have been performed for educational purposes [1] and for the investigation of the formation and propagation of spiral structure using an analytic static potential for the halo [3]. Both of these simulations used $\sim 100$ million particles. Recently Fujii et al. [16] and Sellwood [19] demonstrated that the number of particles adopted in those 
simulations is not sufficient to accurately resolve relaxation processes and tend to overproduce the dynamical heating of the disk. Although they conclude that at least several hundred thousand particles are required to prevent numerical heating, it is still unclear which resolution is required in order to accurately reproduce the intricate non-linear dynamics of the internal structure of a Milky Way sized galaxy. Furthermore, recent observation campaigns such as Gaia require even more accurate simulations with at least 100 times more particles to allow a direct comparison between observations and simulations. This realization has led to the requirement for improving the parallel performance of simulation codes before it becomes possible to simulate the Milky Way Galaxy with sufficient resolution to resolve these issues. With the code described in this work, we can simulate the Galaxy on a star-by-star basis, which is a sufficiently high resolution to address these questions, and may therefore change our understanding of the dynamical evolution of disk galaxies like the Milky Way.

Tree-codes have been used in various incarnations such as TreePM [20]-[27] for previous Gordon Bell Prize simulations [10], [28]-[32]. Recently, Ishiyama et al. [10] achieved 4.45 Pflops average performance on 82944 nodes (663552 CPU cores) of $\mathrm{K}$ computer with a cosmological tree particle-mesh calculation using one trillion particles. Most of the previous tree and TreePM codes have been tuned for massively parallel supercomputers without accelerators [10], [23], [24], [26], [27], [33] except for codes optimized for relatively small clusters including accelerators such as GPUs and GRAPEs [25], [31], [32], [34], [35]. The first calculation with a parallel tree-code with GPUs was running a 1.6 billion particle cosmological dark-matter simulation on 256 GPUs [31]. They achieved a sustained performance of 42 Tflops and won the Gordon Bell Prize with their performance/price ratio of 124 Mflops/\$. The next year the same team [32] received an honorable mention of the Gordon Bell Prize committee for their 190 Tflops cosmological simulation with 3.3 billion particles on 576 GPUs on the DEGIMA supercomputer (at the Nagasaki Advanced Computing Center). Their prize performance ratio was $254.4 \mathrm{Mflops} / \$$. They used the GPUs as gravity accelerators, in very much the same way as the GRAPE [36], [37] family of special purpose computers and the first GPUs [38], [39] have been used [34]. In these calculations the host processors were used for constructing the tree and walking the tree-structure to build interaction lists, and the GPU was used for the force calculations only. In their calculations constructing and walking the tree $(\mathcal{O}(N \log N)$ operations) becomes a bottleneck, as does the communication with the GPU.

The move from CPU-based to GPU-based supercomputers is motivated by lower energy consumption per flop for the latter. For example, K computer offers 830 Mflops/watt compared to 2.1 (2.7) Gflops/watt for Titan (Piz Daint) 1 . We therefore expect that new exascale supercomputers will be equipped with similar type of accelerators (see $\S$ VII . However, on Titan the theoretical peak performance is 3.95 Tflops per node (in single precision and excluding CPU performance), wheareas it is 0.128 Tflops per node on $\mathrm{K}$ computer. The memory and network performance per flop is therefore much lower on Titan than it is on $\mathrm{K}$ computer. While achieving high performance on

\footnotetext{
${ }^{1}$ see http://www.green500.org/
}

the GPU, we increase the need for a high-speed network, which requires an even more aggressive strategy for minimizing the communication cost.

Designing a strategy to minimize the CPU-GPU and network communication will therefore result in a general improvement of a wide variety of algorithms because our optimization strategy is not specific to $N$-body simulations. In our solution we reduced this communication by having the GPU perform the tree construction and tree-walk, as well as the force calculations. The communication between nodes is minimized by using a careful selection strategy of which data we send as well as by reusing this data for multiple purposes. The resulting implementation is not specific to Titan or Piz Daint, but, in fact, can be applied on any large GPU-equipped supercomputer, such as the TSUBAME (at the Tokyo institute of Technology's Global Scientific information Center) series of supercomputers, HA-PACS (at University of Tsukuba), Tianhe1A (National Supercomputing Center of Tianjin), Nebulae (NSCS), PLX-GPU (CINECA) and LGM (Leiden).

The two important improvements compared to earlier work with parallel tree-codes, are: 1) porting the tree-code, including the tree-building and tree-walk, entirely to the GPU and 2) utilizing the node CPU for orchestrating the communication, administrative purposes, feeding the GPU, and data reduction. Improvement 1 allowed us to achieve high performance by efficiently utilizing each GPU and improvement 2 enabled us to scale the code to 18600 nodes even with a rather modest number of particles compared to previous GBP winners.

\section{WHAT THE INNOVATIONS ARE AND HOW THEY WERE ACHIEVED}

Given the enormous computational capabilities of the NVIDIA Kepler GPU, communication between the MPI processes can easily become the major scalability barrier, especially when there are $1000+$ nodes $^{2}$. The code used in this work is our parallel GPU tree code called Bonsai ${ }^{3}$ Here we shortly describe single GPU optimizations for the Kepler architecture and cover in detail the parallelization scheme used to distribute the work across multiple GPUs. For more details about the original single GPU version we refer the reader to our previous work [9].

\section{A. Single GPU Optimizations}

The single GPU code consists of three major parts: treeconstruction, computation of multipole moments, and treewalk in which inter-particle gravitational forces are computed. In contrast to previous works [31], [32], in Bonsai all of these steps are carried out on the GPU, leaving the CPU with lightweight tasks such as data management and kernel launches.

The assimilation of the tree-walk and force computations into a single GPU kernel allows us to achieve excellent computational efficiency by not wasting the GPU's memory bandwidth for saving the particle interaction lists in the off-chip

\footnotetext{
${ }^{2}$ We use one GPU per MPI process.

3 Bonsai is publicly available as part of the AMUSE framework [40] at http://amusecode.org or as standalone tool at https://github. com/treecode/Bonsai
} 


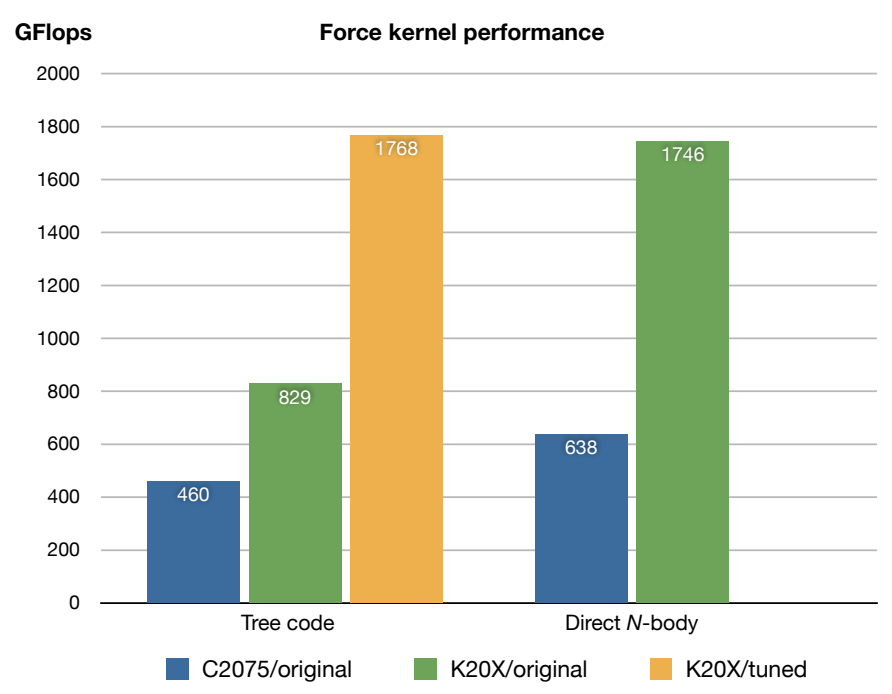

Fig. 1. Performance of the gravitational force kernel. The blue bars indicate the performance of the Fermi kernel on the C2075. The green bars indicate the performance of the Fermi kernel on a K20X. The gold bar shows the performance of the $\mathrm{K} 20 \mathrm{X}$ tuned kernel. With tuning, the $\mathrm{K} 20 \mathrm{X}$ is twice as fast as the original kernel, and is $4 \mathrm{x}$ faster than the $\mathrm{C} 2075$. Also presented here is the performance of the direct $N$-body kernel from NVIDIA's CUDA SDk 5.5 running on the same hardware.

memory. Instead, the interaction lists are stored in registers and evaluated on-the-fly during the tree-walk, therefore delivering superb performance in excess of 1.7 Tflops on a single K20X. The details of the tree-walk on NVIDIA Fermi architecture are described in [9]. A naive use of the Fermi optimized kernels on Kepler GPUs delivers relatively poor performance. In Fig. 1 we show that the Fermi kernels only deliver twice the performance improvement, while the hardware is four times faster in (peak) single precision. The main limitation was caused by excessive use of shared memory. The Kepler architecture introduced shfl intrinsics for intra-warf 4 communication. By taking advantage of these capabilities, we were able to reduce the shared memory usage by $90 \%$ in favour of registers and recover the missing performance.

\section{B. Multi-GPU Parallelization}

Maintaining Bonsai's excellent single-GPU efficiency when scaled to many GPUs requires both the minimization of the amount of data traffic between different GPUs, and hiding the communication steps behind computations. We achieve this by carefully selecting, combining and expanding different well-known parallelization strategies. After experimenting we eventually settled on a combination of the Local Essential Tree (LET) and Space Filling Curve (SFC) methods.

In the original LET method [28], the physical domain is divided into rectangular sub-domains via a recursive multisection algorithm. Each process uses these sub-domains to determine which part of its local data (that is to say which LET) will be required by a remote process. After a process has received all the required LET structures, they are merged into the local tree to compute the gravitational forces. This

\footnotetext{
${ }^{4} \mathrm{~A}$ warp is a group of threads which are executed in lock-step. On current NVIDIA hardware, a warp has 32 threads.
}

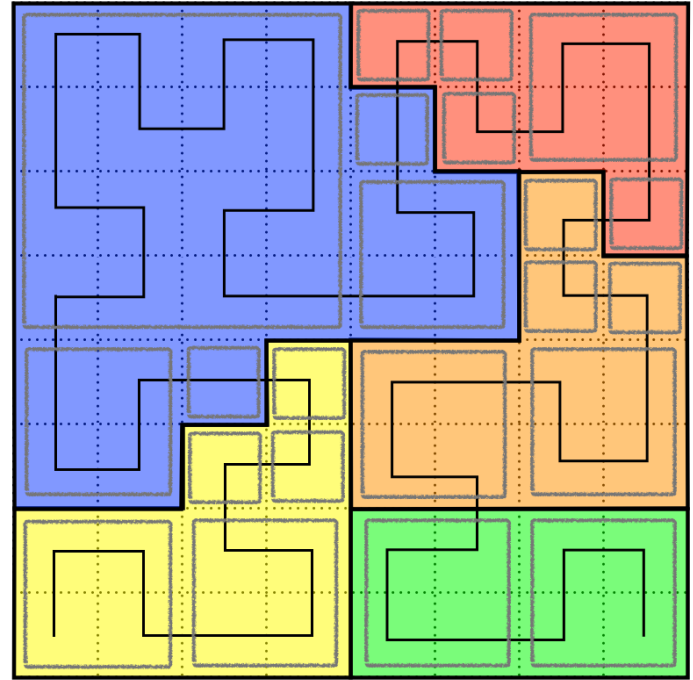

Fig. 2. Example of domain decomposition with a Peano-Hilbert space filling curve (black solid line). The colors indicate the five separate domains. The gray squares correspond to the tree-cells, which consist solely of particles belonging to a single process.

method was used in several award-winning papers [10], [31], [32].

In the SFC method, particles are ordered along an SFC which is split into equal pieces that define the sub-domain boundaries. These boundaries will have a fractal shape, which makes it non-trivial to build a compact LET structure. For example, Fig. 2 illustrates a Peano-Hilbert space filling curve (PH-SFC) [41] and the corresponding decomposition to 5 sub-domains. To avoid these complications, SFC-based tree codes usually do not use LET structures. Rather, they either export particles to remote processes, compute partial forces, and import results back [24], or request sub-trees from the remote processes [42]-[44]. Such methods generate multiple communication steps during the tree-walk.

The LET method requires the least amount of communication. For this reason, and despite the difficulties of dealing with the fractal boundaries, we combine LET with the SFC domain decomposition which guarantees that sub-domain boundaries are branches of a hypothetical global octree. This step allows us to skip merging the imported structures into the local-tree, but rather process them separately as soon as they arrive, thereby hiding the communication behind computations.

1) Domain Decomposition \& Load Balancing: We take the following steps to compute the Peano-Hilbert (PH) keys [41] that form the basis of the tree-construction and domain composition. Each GPU computes its local bounding box, after which the CPUs determine the global bounding box, whose geometric properties are used to map particle coordinates into global PH keys. These keys are used to determine the local domain geometry of each of the processes by means of the parallel sampling method.

In the original sampling method [45], each process samples local keys with a fixed rate, $R$, which are sent to a domaindecomposition process (DD-process) that combines these into a global PH-SFC. This SFC is cut into $p$ equal pieces, whose 
beginning and ending $\mathrm{PH}-\mathrm{key}$ determines the corresponding local domain geometry of individual processes. This method, however, proved to be inadequate for our purpose because as the number of processes increases, and while keeping the average number of particles per process the same, the sampling rate must be increased. The net result is that for a sufficiently large $p$ the domain decomposition becomes a serial bottleneck in the code.

We parallelized the original sampling method by first factoring the number of processes, $p=p_{x} \times p_{y}$, where $p_{x}$ is the number of DD-processes and $p_{y}$ the number of processes handled by each DD-process. Similarly to the original method, each process samples local keys with a fixed sampling rate, $R_{1}$, which are then gathered by a DD-process. However, in contrast to the serial method, the resulting SFC is cut into $p_{x}$ equal pieces, whose boundaries are broadcast. Next, each of the processes samples keys with a different fixed rate, $R_{2}$, and the sampled keys are sorted into each of the $p_{x}$ sub-domains. The keys belonging to each of the sub-domains are gathered by a corresponding DD-process, which combines them into a partial SFC and cuts it into $p_{y}$ equal pieces; in total, $p_{x}$ DDprocesses will have $p_{y}$ pieces each. All these are gathered and merged into the $p=p_{x} \times p_{y}$ pieces by a DD-process. As in the original method, the beginning and ending $\mathrm{PH}$ keys of the pieces determine the new local domain geometry for each of the processes.

Finally, each of the sampling rates includes a correction factor needed to achieve load balance. In particular, we balance the number of floating point operations executed by the GPU tree-walk kernel with the restriction that a process cannot have $30 \%$ more than the average number of particles per GPU [26].

With the domain boundaries at hand, each GPU generates a list of particles that are not part of its local domain, and these particles are then exchanged between the processes. After the particle exchange is complete, each GPU rebuilds its tree-structure and computes the corresponding multipole moments. At the end of this step, each process has all necessary information to proceed with the force calculation. We would like to stress that each local tree is a non-overlapping branch of a hypothetical global octree, because we use the PH-SFC as a basis for the domain decomposition. This guarantees binary consistency of the domain decomposition independent of the number of processes, and allows us to hide LET communication behind computation.

2) Computing the gravity: Due to the long-range nature of Newton's universal law of gravitation [46], the computation of mutual forces is by definition an all-to-all operation. Therefore, to compute forces on local particles, a target process requires communication with all other processes during each iteration. We do this by forcing remote processes to send the required particle and cell data (LETs) to the target process. In order to build a LET structure for a remote domain, a process has to know the physical boundaries of that remote domain.

To extract these boundaries we use the local tree-structure and select the cells that form the edges of the local particle set (gray squares in Fig.2). We then send a copy of our local tree in which all cells except these boundary cells (and their parents) are removed. In this way, we can also use this tree as a LET structure. To gather boundary trees, we use the
MPI_Al lgatherv collective. Once this communication step is complete we continue with the computation and exchange of the actual LET structures. This requires two steps, both of which contain floating point and memory bandwidth intensive operations. In order for the code to scale efficiently, these steps must overlap with the force computation on the GPU. To achieve this we use CPU multi-threading via OpenMP, with which we split each MPI process into three thread-groups: one thread is responsible for MPI communication, which we refer to as the communication thread, another thread drives the GPU, which we call the driver thread, and the rest of the threads are busy computing, which we collectively call the compute threads.

In the first step, the compute threads check whether the boundaries of the remote domain contain enough information to compute the forces for our local particles, or whether a more extensive LET is required from the remote domain. At the same time we also do the same check whether the boundary information that we have sent to the remote domain is indeed sufficient for that domain to compute the gravitational forces, or whether a more extensive LET structure has to be sent. By carrying out the same checks for ourselves and for the remote domain we perform double the amount compute work but this reduces the amount of required communication and increases the asynchronicity of the LET process, as we do not have to wait on a signal from the remote domain. If the boundaries can be used as LET structures, no further communication will be required with the processes for which we use the boundaries. The exceptions are our $\sim 40$ nearest neighbors which require more data than our boundaries can provide and for these processes we have to execute the second step.

In the second step, the compute threads prepare specific LET structures for each of the domains that require more information than what is contained in the boundaries. At the same time, the communication thread is busy sending prepared LET data as it arrives from the compute threads, as well as receiving LET data from remote processes which it passes to the driver thread. The driver thread merges these LET trees into a sub-tree, in which LET structures form branches. In other words, this reconstructs the necessary branches of the hypothetical global octree on the fly, but only using LET data currently received from remote processes. Whenever the GPU is ready with the gravitational force kernel, either on the local data or the already received remote data, the newly built LET tree is fed to the GPU.

Once the gravitational forces are computed for all local particles, we advance them forward in time using a $2^{\text {nd }}$-order leap-frog integration scheme [47]. This marks the end of the $N$-body integration step.

Since the above method is based on the tree-structures, the amount of communication per process during the boundary exchange is virtually independent of the number of particles per GPU, and depends only on the number of processes and the simulated physical model. Furthermore, the gravity step as a whole becomes more efficient with more particles per GPU because the time window to hide the communication becomes larger. The communication time itself increases only slightly because the number of particles at the domain surface, which are commonly used for the communication, increases 
at a lower rate than the total number of particles inside the domain volume.

\section{ApPliCATION AND EARLy SCIENTIFIC RESUlTS}

The Milky Way model that we use contains a central bulge and stellar disk which are embedded inside a dark matter halo. In this model [48] the halo has a mass of $6.0 \times 10^{11} M_{\odot}$ and uses an Navarro-Frenk-White (NFW) density distribution [49]. The stellar disk has a mass of $5.0 \times 10^{10} M_{\odot}$ and follows an exponential distribution while the bulge stars are based on a Hernquist density profile [50] with a total mass of $4.6 \times$ $10^{9} M_{\odot}$. We realized this model with a total of $51,199,967,232$ (51 billion) particles, divided over the bulge, disk, and halo with 994,689,024 (1 billion), 2,945,105,920 (3 billion), and 47,260,172,288 (47 billion) particles respectively. We adopt equal masses for each of the particles for all three components in order to avoid numerical heating caused by unequal mass. This results in a mass resolution of $\sim 10 M_{\odot}$, and a spatial resolution of 1 parsec 5 (pc).

This simulation has a particle resolution in the bulge and disk 200 times greather than the previous highest resolution simulation of the Milky Way [5]. The halo is resolved with 500 and 5000 times more particles compared to [5] and [14] respectively. To generate the initial conditions (IC) for the Milky Way model, we use GalacticICS [51] which creates a smooth exponential disk, and a spherically symmetric bulge and halo. Given the sheer size of the initial model, it is crucial to modify this IC generator for distributed and multi-threaded execution. Furthermore, to avoid heavy start-up I/O related to reading large IC files, we decided to generate all our Milky Way models on the fly.

The large number of particles enables us to adopt a smaller softening length, which results in significantly higher spatial resolution compared to previous simulations; in contrast to mass resolution, spatial resolution scales as $\mathcal{O}\left(N^{1 / 3}\right)$. We adopt a softening length of $1 \mathrm{pc}$, which is an order of magnitude smaller than in previous simulations [5], [14]. Furthermore most of the previous simulations adopted an opening angle for the tree-code of $\theta=0.7$, which is acceptable for spheroidal systems, but to accurately model irregular smallscale structures, such as spiral arms, a significantly smaller opening angle is required. Therefore we used $\theta=0.4$ even though this increases the calculation costs proportionally to $\mathcal{O}\left(\theta^{-3}\right)[52]$. We simulated this Milky Way model for 6 billion years, at which point spiral arms and a bar structure have fully formed.

In the top panels of Fig. 3 we present the time evolution of the face-on surface density of the disk and bulge. The galaxy did not form any prominent structure up to half-way through the simulation ( $\sim 3$ billion year). Shortly afterward, however, a bar structure formed which then induced the formation of spiral arms. At $t \sim 5$ billion years, a barred spiral galaxy similar to the Milky Way has formed. The time at which the bar and spiral structure forms appears to increase with the number of particles, and therefore a longer simulation time may be required for larger models. The bottom right panel of Fig. 3 is a zoom-in of the area around where the Solar System is located, $8 \mathrm{kpc}(\sim 26,000$ light-year) from the Galactic Center.

\footnotetext{
${ }^{5} 1$ parsec is 3.26 light year.
}

The high resolution of the simulations allows us to resolve the fine structure of the spiral arms and the results can be directly compared to observations.

In the bottom left panel of Fig. 3, we present the velocity structure near the Sun, which is the region marked by the circle in the bottom middle panel. Several streams and spots of high density regions can be observed. These are also known as 'moving groups', and many similar structures have been found in recent observations of the stars in the Solar Neighborhood [53]. These moving group structures are considered to be formed by resonances originating from the bar [54]; however, also the spiral arms are capable of forming such velocity structures [55]. Comparing our simulation data with observational data allows us to study the dynamics of the bar and the spiral structures in the Milky Way. With this simulation we are able, for the first time, to obtain enough sample particles $(68,000$ within $500 \mathrm{pc})$ from a self-consistent simulation to make a direct comparison with observations that contain a comparable number of observed stars (57,000 within 200-300 pc) [53].

At the time of writing, observations have only provided kinematic data for the Milk Way disk stars that are located in the Solar Neighborhood [53]. However, the future Gaia catalogue will extend this to stars beyond the Solar Neighborhood, and comparing these observations against our simulations will provide us with further details of the structure of the Milky Way Galaxy such as the size and the pattern speed of the bar, the structure of the spiral arms, and the resonance effects in the velocity distribution.

In addition, a more detailed analysis of the currently simulated galaxy will provide us with numerous pieces of information that can point us to the origin of the observed structures in the Milky Way and how it has evolved during the 10 billion years that it took to form the current-day Milky Way.

\section{SYSTEM AND ENVIRONMENT WHERE PERFORMANCE WAS MEASURED}

The Bonsai tree-code we use to perform our Milky Way simulations is optimized to run on the NVIDIA Fermi and Kepler architectures (see $\S$ III). The highest performance is obtained on the K20X, because of the hardware improvements introduced with the Kepler generation GPUs. The code operates on distributed memory systems with one or more GPUs per node.

For the performance measurements and production runs in this work we used two machines. The first is the Swiss Cray XC30 machine, Piz Daint, which has a total of 5272 nodes. The second is the Cray XK7 supercomputer, Titan, at Oak Ridge National Laboratory in the USA which has a total of 18688 nodes (see Table II for details). Although both these machines are designed by Cray, they have different interconnect and CPUs. Piz Daint is the newer of the two supercomputers, and is equipped with Intel CPUs as well as a more advanced network generation, Aries, where the nodes are connected in a dragonfly topology. Titan, on the other hand, contains AMD CPUs and the nodes are connected using the Gemini network architecture which uses a 3D torus topology. The faster CPUs and much improved interconnect of Piz Daint benefit the 

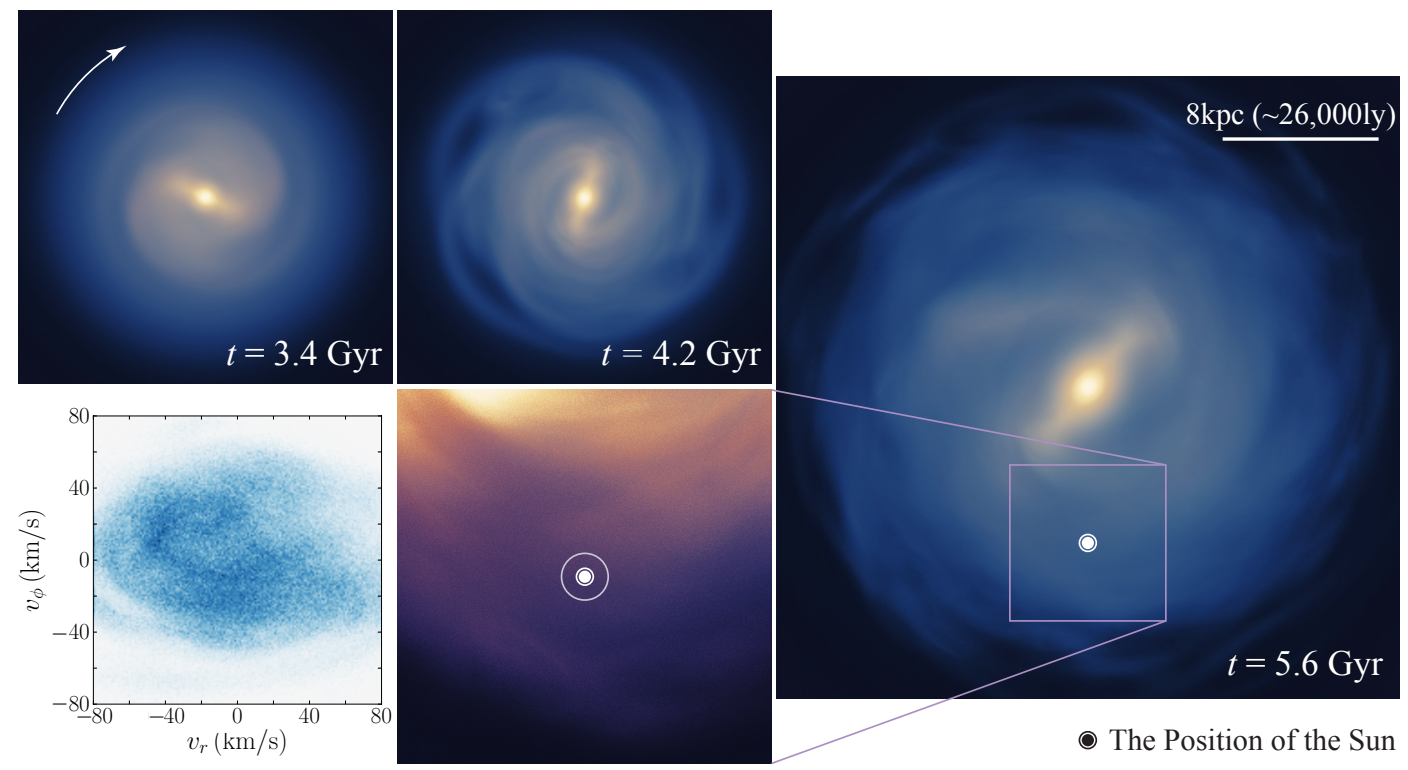

Fig. 3. Face-on surface density of the Galactic disk and the bulge from the 51 billion particle Milky Way simulation. The two top panels show the density at 3.4 and 4.2 billion years, and the arrow in the left panel shows the rotation direction of the Galactic disk. The large rightmost panel shows density plot at 5.6 billion years. The bottom right panel is a zoom-in of the spiral region marked in the rightmost panel, where the Sun in the Milky Way is thought to be located ( $8 \mathrm{kpc}$ from the Galactic Center). The bottom left panel is the radial and azimuthal velocity distribution of the stars within a $500 \mathrm{pc}$ radius centered around the position of the Sun (circle marker in the bottom middle panel), in which the rotation velocity of the disk is subtracted from the azimuthal velocity.

parallel performance of Bonsai during the communication phase as we will see in $\S \mathrm{VI}$.

Bonsai runs on any computer as long as it contains an NVIDIA GPU with CUDA support. This includes laptops, desktops and clusters. The number of particles that can be used per GPU depends on the amount of memory available to the GPU. Both the Fermi and Kepler architectures are supported. For single GPU simulations the only CPU communication required involves kernel launches and the retrieval of results from the GPU. When using multiple GPUs the speed of the CPU, PCI-E bus and network become crucial to achieve high performance as we have to communicate between all the MPI processes. The CPU cores are used for writing result data to disk, network communication, preparing LET data to be sent over the network, receiving LET data that is sent to us by a remote process, and for feeding the GPU. The GPU handles the data processing, force computations, particle integration and creates the tree-structures for the local data.

\section{Performance ReSUlts}

We present the performance results by calculating floating point operations for only the force calculations. We ignore contributions from tree construction, computation of multipole moments, multipole acceptance criteria during the tree-walk, all LET-related operations (which are performed on the CPU), time integration and diagnostics. The timing of these operations however, is taken into account when we calculate the application performance.
TABLE I. HARDWARE USED FOR OUR PARALLEL SIMULATIONS. ON BOTH SYSTEMS WE USED CUDA 5.5, GCC 4.8.2 AND THE CRAY MPICH 6.2 LIBRARY.

\begin{tabular}{|lcc|}
\hline Setup & Piz Daint & Titan \\
\hline GPU model & K20X & K20X \\
GPU/node & 1 & 1 \\
Total GPUs & 5272 & 18688 \\
GPUs used & 5200 & 18600 \\
GPU RAM (ECC enabled) & $5.4 \mathrm{~GB}$ & $5.4 \mathrm{~GB}$ \\
\hline CPU model & Xeon E5-2670 & Opteron 6274 \\
CPU/node & 1 & 1 \\
Total CPUs & 5272 & 18688 \\
CPUs used & 5200 & 18600 \\
CPU cores used & 41600 & 297600 \\
Node RAM & $32 \mathrm{~GB}$ & $32 \mathrm{~GB}$ \\
\hline Network & Cray Aries/dragonfly & Cray Gemini/3D Torus \\
\hline
\end{tabular}

\section{A. Operation counts}

The accelerations, $\boldsymbol{a}_{i}$, and potential, $\phi_{i}$, of particle $i$, which we collectively call the force, are computed by

$$
\begin{aligned}
& \phi_{i}=\sum_{j} {\left[-\frac{m_{j}}{\left|\boldsymbol{r}_{i j}\right|}+\frac{1}{2} \frac{\operatorname{tr}\left(\mathbf{Q}_{j}\right)}{\left|\boldsymbol{r}_{i j}\right|^{3}}-\frac{3}{2} \frac{\boldsymbol{r}_{i j}^{T} \mathbf{Q}_{j} \boldsymbol{r}_{i j}}{\left|\boldsymbol{r}_{i j}\right|^{5}}\right], } \\
& \boldsymbol{a}_{i}=\sum_{j}\left[\frac{m_{j} \boldsymbol{r}_{i j}}{\left|\boldsymbol{r}_{i j}\right|^{3}}-\frac{3}{2} \frac{\operatorname{tr}\left(\mathbf{Q}_{j}\right) \boldsymbol{r}_{i j}}{\left|\boldsymbol{r}_{i j}\right|^{5}}\right. \\
&\left.-\frac{3 \mathbf{Q}_{j} \boldsymbol{r}_{i j}}{\left|\boldsymbol{r}_{i j}\right|^{5}}+\frac{15}{2} \frac{\left(\boldsymbol{r}_{i j}^{T} \mathbf{Q}_{j} \boldsymbol{r}_{i j}\right) \boldsymbol{r}_{i j}}{\left|\boldsymbol{r}_{i j}\right|^{7}}\right],
\end{aligned}
$$

where $\boldsymbol{r}_{i j}=\boldsymbol{r}_{j}-\boldsymbol{r}_{i}$. Here, $m_{j}, \boldsymbol{r}_{j}, \mathbf{Q}_{j}$ are mass, position and quadrupole moments (in a $3 \times 3$ symmetric matrix) of particle $j$ respectively. Computation of one particle-particle interaction ( $\mathrm{p}-\mathrm{p}$ ) without the quadrupole moment term consists of 4 subtraction (sub), 3 multiplication (mul), 6 fused-multiply- 
add (fma), and 1 reciprocal-square-root (rsqrt) instructions. In this article, we count 4 floating-point operations for the reciprocal-square-root, which results in a total of $\mathbf{2 3}$ operations for each p-p. A particle-cell interaction (p-c) with quadrupole corrections consists of 4 sub, 6 add, 17 mul, 17 fma and 1 rsqrt, which results in 65 operations for $\mathrm{p}$-c interaction 6 The total number of flops is obtained by multiplying these numbers by the total number of $\mathrm{p}-\mathrm{p}$ and $\mathrm{p}-\mathrm{c}$ (as recorded during execution), and divided by the execution time.

Note that [28]-[32] used $\mathbf{3 8}$ for the operation count of a $p-p$ interaction. Although it is convenient to use the same operation count for comparing one record with the other, this can over count the operations for hardware with fast rsqrt support. Previous GBP winner [10] counted 51, within which about half was spent in the calculation of a cut-off polynomial.

\section{B. Scalability}

To evaluate the scalability and parallel performance of Bonsai we conduct both weak and strong scaling studies. In both studies we use the Milky Way Galaxy model as initial condition (see $\S \mathrm{IV}$ ), the model was integrated for 64 timesteps. The measurements to assess code scalability were taken from time-steps 32 until 64, which allowed the simulation to relax into a load-balanced state. This is a good representation of long-term integrations, in which significant changes to the physical configuration take place on a time-scale of hundreds of iterations.

In the weak scaling test we used on average 13 million particles per GPU, which is comparable to the number of particles we used in the production runs. It is possible to do runs with up to 20 million particles per K20X, and thereby achieve higher application performance, as more time is spent on the GPU, but that is not comparable to the production run configurations that we use. For all the performance measurements and production simulations we used an opening angle of $\theta=0.4$, which is satisfactory to properly model disk galaxies, such as the Milky Way (see $\S[\mathrm{IV}$ ).

In Fig. 4 we show the weak scaling and parallel efficiency for simulations on both Titan and Piz Daint. On Piz Daint we used between 1 and 5200 GPUs and on Titan we used between 1 and 18600 GPUs. The parallel efficiency of the simulations on Piz Daint never drops below 95\%, indicating that most of the required communication is hidden behind the GPU work. On Titan the parallel efficiency for the simulations up to 8192 GPUs is around $90 \%$. This is slightly lower than on Piz Daint and is caused by a combination of the slightly higher communication times on the older network and slightly longer LET generation times because of the slower AMD CPUs. The breakdown of these timings can be found in Tab. II] For the largest run on Titan, with 18600 nodes and a total of 242 billion particles, we reach a GPU single precision performance of 33.49 Pflops and a sustained application performance of 24.77 Pflops. This results in a parallel efficiency of $86 \%$ when compared to a single GPU. The drop in efficiency when compared to 8192 nodes is due to both longer network communication times and increased imbalance. Finally, on

\footnotetext{
${ }^{6}$ All operation counts were verified with the disassembling command cuobjdump -sass in the CUDA toolkit.
}

18600 nodes with 242 billion particles in total, the average wall-clock time of a full time-step is 4.8 seconds.

We also demonstrate the strong scaling behavior of Bonsai in Tab. II. In particular, on Piz Daint we simulated a 26.6 billion particle Milky Way model, while on Titan we simulated a model two times larger. In both cases, we find satisfactory strong scaling, with a parallel efficiency of $95 \%$ on Piz Daint when scaled from 2048 to 4096 GPUs and $87 \%$ on Titan when scaled from 4096 to 8192 GPUs.

\section{Time-to-solution}

Our production simulation of the Milky Way Galaxy for 6 billion years with a 51 billion particle model was carried out on 4096 GPU nodes of Piz Daint. With this simulation we were able, for the first time, to increase the spatial accuracy by an order of magnitude compared to previous state-of-the-art simulations. We achieved this by using over 1000 times more particles than before, but we also tripled the time evolution of the simulation up to 6 billion years.

After the bar structure and spiral arms are fully formed we measure the simulation time for 1000 iterations; the time-step duration is determined as the average of those 1000 iterations. We measure after the formation of the bar and spiral arms because the additional small-scale structure results in regions with higher density than the mean density of the model, and it is not uncommon for gravitational tree-codes to increase the interaction count in such regions, which negatively impacts the runtime. Indeed, the average calculation time for an iteration at $T=3.8$ billion year is 4.6 seconds, which is about $10 \%$ larger than at the beginning of the simulation. In addition, there was a few percent I/O-related overhead related to storing intermediate simulation snapshots (for the dual purpose of restarting and detailed analysis), as well as rudimentary onthe-fly data analysis.

Using this data we can estimate the wall-clock time needed to simulate the Milky Way Galaxy with a 242 billion particle model on 18600 GPUs. With a softening of 1 parsec, the minimal time step required for an accurate simulation is 75,000 year (this corresponds to the time that two particles pass each other within a softening length). This softening length was also used in our earlier calculations [13], and is considerably smaller than typically used in other Galaxy simulations [2], [5], [16]. If we wish to simulate the evolution of the Milky Way Galaxy for $8 \mathrm{Gyr}$, with a time step of $0.075 \mathrm{Myr}$ we require about 106,667 time steps. With an expected maximum of about 5.5 seconds per time step for a Milky Model with a central bar and fully formed spiral arms, it will take at most a week of computing time. For a more modest model with 106 billion particles using 8192 nodes the maximum expected calculation time per step will be 5.1 seconds and a full simulation, using the above settings, would take just over six days.

\section{Peak performance}

We achieved a sustained application single precision performance of 24.77 Pflops on a 242-billion-particle Milky Way model with 18600 GPUs. When busy with the force computation, the GPUs were processing at an aggregated rate of 33.49 Pflops. This translates to 1.8 Tflops per GPU and 1.33 


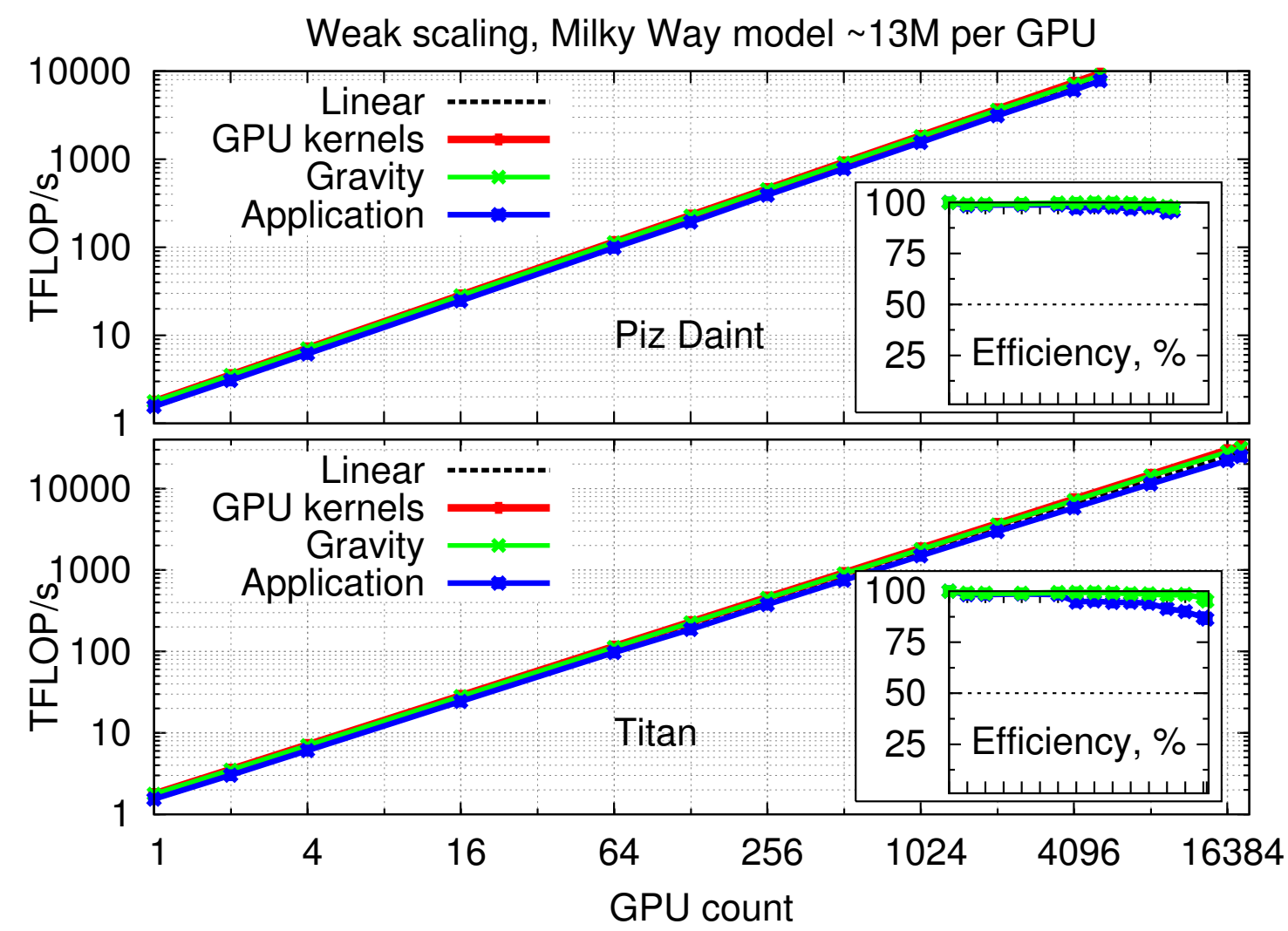

Fig. 4. Weak scaling performance for Piz Daint and Titan. For both panels we used simulations of our Milky Way model with on average 13 million particles per GPU and $\theta=0.4$ as the opening angle. The x-axis of the panels indicates the number of GPUs used and the y-axis the performance in Tflops. The red solid lines show the performance of the tree-walk kernel running on the GPU. The green solid lines show the performance of the gravity step, including the time to communicate LET structures. The blue solid lines show the performance of the full application. The black dashed lines, which are mostly hidden behind the blue lines, indicate linear scaling The lower right insets shows parallel efficiency of the code when compared to a single node. In these insets the green line indicates the efficiency of the gravity step, including communication. The blue line indicates parallel application efficiency with respect to a single GPU. The top panel shows the performance of the simulations on Piz Daint for 1 to 5200 GPUs. The bottom panel shows the performance of the simulations on Titan for 1 to 18600 GPUs.

Tflops for the overall application performance per node. The theoretical peak single precision performance of $18600 \mathrm{~K} 20 \mathrm{X}$ GPUs is 73.2 Pflops. During the force calculation the GPUs operate at $46 \%$ of this number, while the overall application reached $34 \%$ of this peak performance.

\section{IMPLICATIONS FOR FUTURE SYSTEMS AND APPLICATIONS}

The combination of high-performance accelerators and multi-core processors in a single compute node is one of the important trends in high performance computing. At the time of writing, nearly $90 \%$ of the peak performance is due to accelerators in the two fastest supercomputers in the world. It is not unreasonable to expect that future large-scale scientific simulations will be tailored to run on such hybrid architectures.

The simulations of the Milky Way Galaxy on the Titan and Piz Daint supercomputers, which we present in this paper, clearly demonstrate that hybrid architectures are well suited for scientific calculations. However, to achieve this goal we had to completely redesign our $N$-body tree-code to fully take advantage of the high-performance network and parallelism offered by GPUs and CPUs.
Much of our efforts in writing Bonsai went into multiGPU parallelization. Instead of letting the CPUs sit idle, we utilized its processing power for the work required to minimize the amount of communication, which in turn permitted us to hide most of the communication behind GPU computations. This can be favorable compared to IBM's BG/Q system with the dedicated communication core: the CPU was in the role of the dedicated communication hardware, the GPU was in the role of the processor doing actual calculations, and with several clever techniques we were able to ensure that both were carrying out their work as concurrently as possible.

With future systems moving toward exascale computing, communication is expected to continue to limit performance. Vendors have already begun to adopt Unified Memory Architectures and Direct Memory Access inside the compute nodes to reduce the communication pressure during these data transfers. This, however, does little to reduce limitations due to interconnect latencies and bandwidth. With intra-node and onchip bandwidths being an order of magnitude larger than that of interconnects, careful attention should be paid to minimize communication. In Bonsai we accomplish this by reducing the number of MPI calls by switching to a push instead of pull method, and using the boundary data for creating LET 


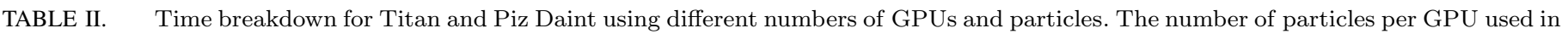
the simulations is indicated by the first row of the table. For each simulation we use our Milky Way model as input data and $\theta=0.4$. The data presented shows the major parts of the algorithm and by which computation unit they are primarily executed. For the first set of rows the first

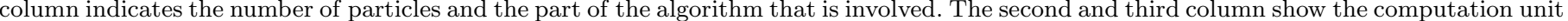
which is involved in this part of the algorithm. The following columns show the execution time, where the first column shows the single GPU timings, the following five the timings on Titan and the final four the timings on Piz Daint. Note that the fifth and fourth columns of the Titan and Piz Daint data respectively shows runs with fewer particles to present the strong scaling data. The 'Non-hidden LET comm' row indicates the part of the communication time during the gravity step that was not hidden behind the GPU work. The 'Other' data includes the time to allocate memory, gather and write statistics, integrate particles and any waiting times induced by load-imbalance. The second set of two rows presents the average number of particle-particle and particle-cell interactions as recorded during the simulations. The bottom two rows give the resulting performance in Tflops for the GPU kernels (excluding communication) and the application as a whole. For Titan we present the weak scaling results for 1024, 2048, 4096 and 18600 GPUs and the strong scaling data for 4096 and 8192 GPUs. For Piz Daint we present the weak scaling results for 1024, 2048 and 4096 GPUs and the strong scaling data for 2048 and 4096 GPUs.

\begin{tabular}{|c|c|c|c|c|c|c|c|c|c|c|c|c|}
\hline \multirow[t]{2}{*}{ Operation } & \multicolumn{2}{|c|}{ Compute unit } & time $[\mathrm{s}]$ & \multicolumn{5}{|c|}{ wall-clock time Titan [s] } & \multicolumn{4}{|c|}{ wall-clock Time Piz Daint [s] } \\
\hline & $\mathrm{CPU}$ & GPU & $1 \mathrm{GPU}$ & 1024 & 2048 & 4096 & 18600 & 8192 & 1024 & 2048 & 4096 & 4096 \\
\hline Nparticles/GPU [Million] & - & - & 13 & 13 & 13 & 13 & 13 & 6.5 & 13 & 13 & 13 & 6.5 \\
\hline Sorting SFC & - & $\mathrm{X}$ & 0.1 & 0.1 & 0.1 & 0.1 & 0.13 & 0.06 & 0.1 & 0.10 & 0.1 & 0.05 \\
\hline Domain Update & $\mathrm{X}$ & - & - & 0.2 & 0.2 & 0.2 & 0.3 & 0.15 & 0.1 & 0.10 & 0.1 & 0.07 \\
\hline Tree-construction & - & $\mathrm{X}$ & 0.11 & 0.1 & 0.1 & 0.1 & 0.1 & 0.05 & 0.1 & 0.10 & 0.1 & 0.05 \\
\hline Tree-properties & - & $\mathrm{X}$ & 0.03 & 0.03 & 0.03 & 0.036 & 0.03 & 0.016 & 0.03 & 0.03 & 0.03 & 0.016 \\
\hline Compute gravity Local-tree & - & $\mathrm{X}$ & 2.45 & 1.45 & 1.45 & 1.45 & 1.45 & 0.68 & 1.45 & 1.45 & 1.45 & 0.68 \\
\hline Compute gravity LETs & - & $\mathrm{X}$ & - & 1.78 & 1.89 & 2.0 & 2.09 & 1.13 & 1.79 & 1.89 & 2.02 & 1.01 \\
\hline Non-hidden LET comm & $\mathrm{X}$ & $\mathrm{X}$ & 一 & 0.09 & 0.1 & 0.14 & 0.22 & 0.25 & 0.09 & 0.06 & 0.07 & 0.07 \\
\hline Unbalance + Other & $\mathrm{X}$ & $\mathrm{X}$ & 0.1 & 0.27 & 0.28 & 0.40 & 0.45 & 0.31 & 0.22 & 0.21 & 0.28 & 0.15 \\
\hline Total & & & 2.79 & 4.02 & 4.15 & 4.41 & 4.77 & 2.65 & 3.84 & 3.94 & 4.15 & 2.1 \\
\hline Interaction type & \multicolumn{12}{|c|}{ interaction count per particle } \\
\hline Particle-Particle & & & 1745 & 1715 & 1716 & 1718 & 1716 & 1716 & 1716 & 1716 & 1718 & 1714 \\
\hline Particle-Cell & & & 4529 & 6287 & 6527 & 6765 & 6920 & 7096 & 6290 & 6515 & 6810 & 6616 \\
\hline Performance [Tflops] & & & K20X & \multicolumn{5}{|c|}{ Titan } & \multicolumn{4}{|c|}{ Piz Daint } \\
\hline GPU & & & 1.77 & 1844.6 & 3693.7 & 7396.8 & 33490 & 14714 & 1844.7 & 3693.9 & 7396.9 & 7383.5 \\
\hline Application & & & 1.55 & 1484.6 & 2971.8 & 5784.9 & 24773 & 10051 & 1551.9 & 3129.9 & 6180.7 & 5947.9 \\
\hline
\end{tabular}

structures and for computing forces. With the increase of the GPU on-board memory (e.g. 12GB on a K40 card), we will be able to store more data on the device, which decreases the relative amount of data that needs to be communicated between the GPUs, thereby having more possibilities to hide the communication time behind computations. Without major improvement of the network latencies and bandwidth in future hardware this will likely be the only option to maintain scalability.

With future techniques, such as the recently announced NVIDIA's NVLINK technology, it will be possible to have much faster communication between GPUs in the same physical node. For Bonsai this could mean that by careful placement of the MPI ranks we can communicate with our direct neighbors in particle space using this high speed connection. This, again, will reduce the pressure on the interconnect, because less data will have to be communicated between nodes.

The large number of cores on modern day CPUs could enable us to incorporate more physics in future simulations. Currently we use as many the CPU cores as possible for the generation of LET structures, but in the future we can reserve some of the cores for other tasks. The galaxy simulations could then be enriched with, for example, stellar evolution and massive black holes with their stellar cusps. The gravitational interactions around the black holes require the accuracy of a direct $N$-body code which, together with the stellar evolution code, would be running on the CPU while the tree-code would be running on the GPU. Such a combination of physics could be realized via the decomposition of physical elements, as is realized in the Astronomical Multi-purpose Software Environment (AMUSE) [56], [57]. In earlier successful attempts the AMUSE environment was used together with heterogeneous hardware to distribute multi-physics simulations across a national network of specialized computers [58].

Our demonstration of the efficiency of running on a massively parallel heterogeneous architecture indicates that hybrid hardware can give excellent performance. Our achieved performance, the good scaling and the short time-to-solution have been realized by a fundamental redesign of the parallel implementation of the Barnes-Hut gravitational tree algorithm. In this method we fully use all the available computing resources and apply them where they are best suited. The GPUs are used for the high throughput parallel methods and the CPUs for the irregular and communication related methods.

\section{ACKNOWLEDGMENTS}

It is our pleasure to thank Jack Wells for arranging direct access on Titan, Thomas Schulthess for arranging direct access on Piz Daint, Arthur Trew and Richard Kenwayand for arranging access to HECToR, all via Directors' Discretion time. We also thank Mark Harris, Stephen Jones and Simon Green from NVIDIA for their assistance in optimizing Bonsai for the NVIDIA K20X. Our gratitude also goes to Daniel Caputo and Alex Rimoldi for their comments and careful proof-reading of the manuscipt and Junichi Baba (ELSI, Tokyo Institute of Technology) for his helpful comments on the scientific results. Part of our calculations were performed on Hector (Edinburgh), HA-PACS (University of Tsukuba), ATERUI (National Astronomical Observatory of Japan) and Little Green Machine (Leiden University). This work was supported by the Netherlands Research Council NWO (grants \#639.073.803 [VICI], \#643.000.802 [JB], \#614.061.608 [AMUSE] and \#612.071.305 [LGM]), by the Netherlands Research School for Astronomy (NOVA), NAOJ Fellow, MEXT HPCI strategic program and KAKENHI (under Grant Number 24740115). This research 
used resources of the Oak Ridge Leadership Computing Facility at the Oak Ridge National Laboratory, which is supported by the Office of Science of the U.S. Department of Energy under Contract No. DE-AC05-00OR22725. This work was supported by a grant from the Swiss National Supercomputing Centre (CSCS) under project ID d24.

\section{REFERENCES}

[1] J. Dubinski, "Visualizing astrophysical N-body systems," New Journal of Physics, vol. 10, no. 12, p. 125002, Dec. 2008.

[2] J. Dubinski, I. Berentzen, and I. Shlosman, "Anatomy of the Bar Instability in Cuspy Dark Matter Halos,” ApJ , vol. 697, pp. 293-310, May 2009.

[3] E. D'Onghia, M. Vogelsberger, and L. Hernquist, "Self-perpetuating Spiral Arms in Disk Galaxies," ApJ , vol. 766, p. 34, Mar. 2013.

[4] J. A. Sellwood and R. G. Carlberg, "Transient Spirals as Superposed Instabilities," ApJ , vol. 785, p. 137, Apr. 2014.

[5] P. Di Matteo, M. Haywood, A. Gomez, L. van Damme, F. Combes, A. Halle, B. Semelin, M. D. Lehnert, and D. Katz, "Mapping a stellar disk into a boxy bulge: The outside-in part of the Milky Way bulge formation," ArXiv e-prints, Apr. 2014.

[6] D. Heggie and P. Hut, The Gravitational Million-Body Problem: A Multidisciplinary Approach to Star Cluster Dynamics. Cambridge University Press, 2003. [Online]. Available: http://books.google.nl/ books?id=dQH7NJRhCvMC

[7] S. Portegies Zwart, "A multi-scale approach to simulate the Galaxy," in 370 Years of Astronomy in Utrecht, ser. Astronomical Society of the Pacific Conference Series, G. Pugliese, A. de Koter, and M. Wijburg, Eds., vol. 470, Jan. 2013, p. 353.

[8] J. Barnes and P. Hut, "A Hierarchical $\mathrm{O}(\mathrm{N} \log \mathrm{N})$ Force-Calculation Algorithm," Nat , vol. 324, pp. 446-449, Dec. 1986.

[9] J. Bédorf, E. Gaburov, and S. Portegies Zwart, "A sparse octree gravitational N-body code that runs entirely on the GPU processor," Journal of Computational Physics, vol. 231, pp. 2825-2839, Apr. 2012.

[10] T. Ishiyama, K. Nitadori, and J. Makino, " 4.45 pflops astrophysical n-body simulation on $\mathrm{k}$ computer: The gravitational trillion-body problem," in Proceedings of the International Conference on High Performance Computing, Networking, Storage and Analysis, ser. SC '12. Los Alamitos, CA, USA: IEEE Computer Society Press, 2012, pp. 5:1-5:10. [Online]. Available: http://dl.acm.org/citation.cfm?id= 2388996.2389003

[11] R. W. Hockney and J. W. Eastwood, Computer Simulation Using Particles. McGraw-Hill, 1981.

[12] G. Besla, N. Kallivayalil, L. Hernquist, R. P. van der Marel, T. J. Cox, and D. Kereš, "Simulations of the Magellanic Stream in a First Infal Scenario," ApJL, vol. 721, pp. L97-L101, Oct. 2010.

[13] J. Bédorf and S. Portegies Zwart, "The effect of many minor mergers on the size growth of compact quiescent galaxies," MNRAS, vol. 431, pp. 767-780, May 2013.

[14] J. Dubinski and D. Chakrabarty, "Warps and Bars from the External Tidal Torques of Tumbling Dark Halos," ApJ , vol. 703, pp. 2068-2081, Oct. 2009.

[15] E. Athanassoula, "Manifold-driven spirals in N-body barred galaxy simulations," MNRAS, vol. 426, pp. L46-L50, Oct. 2012.

[16] M. S. Fujii, J. Baba, T. R. Saitoh, J. Makino, E. Kokubo, and K. Wada, "The Dynamics of Spiral Arms in Pure Stellar Disks," ApJ, vol. 730, p. 109, Apr. 2011.

[17] J. Baba, T. R. Saitoh, and K. Wada, "Dynamics of Non-steady Spiral Arms in Disk Galaxies," ApJ, vol. 763, p. 46, Jan. 2013.

[18] R. J. J. Grand, D. Kawata, and M. Cropper, "Spiral arm pitch angle and galactic shear rate in N-body simulations of disc galaxies," $A \& A$ , vol. 553, p. A77, May 2013.

[19] J. A. Sellwood, "Relaxation in N-body Simulations of Disk Galaxies," ApJL, vol. 769, p. L24, Jun. 2013.

[20] G. Xu, "A New Parallel N-Body Gravity Solver: TPM," ApJS , vol. 98, p. 355, May 1995.
[21] P. Bode, J. P. Ostriker, and G. Xu, "The Tree Particle-Mesh N-Body Gravity Solver," ApJS , vol. 128, pp. 561-569, Jun. 2000.

[22] J. S. Bagla, "TreePM: A Code for Cosmological N-Body Simulations," Journal of Astrophysics and Astronomy, vol. 23, pp. 185-196, Dec. 2002.

[23] J. Dubinski, J. Kim, C. Park, and R. Humble, "GOTPM: a parallel hybrid particle-mesh treecode," New Astron., vol. 9, pp. 111-126, Feb. 2004.

[24] V. Springel, "The cosmological simulation code GADGET-2," MNRAS , vol. 364, pp. 1105-1134, Dec. 2005.

[25] K. Yoshikawa and T. Fukushige, "PPPM and TreePM Methods on GRAPE Systems for Cosmological N-Body Simulations," Publ. Astr. Soc. Japan, vol. 57, pp. 849-860, Dec. 2005.

[26] T. Ishiyama, T. Fukushige, and J. Makino, "GreeM: Massively Parallel TreePM Code for Large Cosmological N -body Simulations," Publ. Astr. Soc. Japan, vol. 61, pp. 1319-, Dec. 2009.

[27] S. Portegies Zwart, T. Ishiyama, D. Groen, K. Nitadori, J. Makino, C. de Laat, S. McMillan, K. Hiraki, S. Harfst, and P. Grosso, "Simulating the universe on an intercontinental grid of supercomputers," IEEE Computer, v.43, No.8, p.63-70, vol. 43, pp. 63-70, Oct. 2010.

[28] M. S. Warren and J. K. Salmon, "Astrophysical n-body simulations using hierarchical tree data structures," in Proceedings of the 1992 ACM/IEEE Conference on Supercomputing, ser. Supercomputing '92. Los Alamitos, CA, USA: IEEE Computer Society Press, 1992, pp. 570-576. [Online]. Available: http://dl.acm.org/citation.cfm?id= 147877.148090

[29] M. S. Warren, T. C. Germann, P. S. Lomdahl, D. M. Beazley, and J. K. Salmon, "Avalon: An alpha/linux cluster achieves 10 gflops for \$15k," in Proceedings of the 1998 ACM/IEEE Conference on Supercomputing, ser. Supercomputing '98. Washington, DC, USA: IEEE Computer Society, 1998, pp. 1-11. [Online]. Available: http://dl.acm.org/citation.cfm?id=509058.509130

[30] A. Kawai, T. Fukushige, and J. Makino, "\$7.0/mflops astrophysical n-body simulation with treecode on grape-5," in Proceedings of the 1999 ACM/IEEE Conference on Supercomputing, ser. Supercomputing '99. New York, NY, USA: ACM, 1999. [Online]. Available: http://doi.acm.org/10.1145/331532.331598

[31] T. Hamada, T. Narumi, R. Yokota, K. Yasuoka, K. Nitadori, and M. Taiji, "42 tflops hierarchical n-body simulations on gpus with applications in both astrophysics and turbulence," in Proceedings of the Conference on High Performance Computing Networking, Storage and Analysis, ser. SC '09. New York, NY, USA: ACM, 2009, pp. 62:1-62:12. [Online]. Available: http://doi.acm.org/10.1145/1654059.1654123

[32] T. Hamada and K. Nitadori, "190 tflops astrophysical n-body simulation on a cluster of gpus," in Proceedings of the 2010 ACM/IEEE International Conference for High Performance Computing, Networking, Storage and Analysis, ser. SC '10. Washington, DC, USA: IEEE Computer Society, 2010, pp. 1-9. [Online]. Available: http://dx.doi.org/10.1109/SC.2010.1

[33] M. S. Warren and J. K. Salmon, "FOREST: A Parallel Treecode for Gravitational N-Body Simulations with up to 20 Million Particles," in Bulletin of the American Astronomical Society, ser. Bulletin of the American Astronomical Society, vol. 23, Sep. 1991, p. 1345.

[34] J. Makino, "A Fast Parallel Treecode with GRAPE," Publications of the Astronomical Society of Japan, vol. 56, pp. 521-531, Jun. 2004.

[35] N. Nakasato, G. Ogiya, Y. Miki, M. Mori, and K. Nomoto, "Astrophysical Particle Simulations on Heterogeneous CPU-GPU Systems," ArXiv e-prints, Jun. 2012.

[36] D. Sugimoto, Y. Chikada, J. Makino, T. Ito, T. Ebisuzaki, and M. Umemura, "A special-purpose computer for gravitational many-body problems," Nat, vol. 345, pp. 33-35, May 1990.

[37] J. Makino and M. Taiji, Scientific simulations with special-purpose computers : The GRAPE systems. Scientific simulations with specialpurpose computers : The GRAPE systems /by Junichiro Makino \& Makoto Taiji. Chichester ; Toronto : John Wiley \& Sons, c1998., 1998.

[38] L. Nyland, M. Harris, and J. Prins, "The rapid evaluation of potential fields using programmable graphics hardware," 2004.

[39] S. F. Portegies Zwart, R. G. Belleman, and P. M. Geldof, "High- 
performance direct gravitational N-body simulations on graphics processing units," New Astronomy, vol. 12, pp. 641-650, Nov. 2007.

[40] S. F. Portegies Zwart, S. L. W. McMillan, A. van Elteren, F. I. Pelupessy, and N. de Vries, "Multi-physics simulations using a hierarchical interchangeable software interface," Computer Physics Communications, vol. 184, pp. 456-468, Mar. 2013.

[41] D. Hilbert, "Ueber die stetige abbildung einer line auf ein flächenstück," Mathematische Annalen, vol. 38, no. 3, pp. 459-460, 1891. [Online] Available: http://dx.doi.org/10.1007/bf01199431

[42] M. S. Warren and J. K. Salmon, "A parallel hashed oct-tree n-body algorithm," in Supercomputing '93: Proceedings of the 1993 ACM/IEEE conference on Supercomputing. New York, NY, USA: ACM, 1993, pp. 12-21.

[43] M. Winkel, R. Speck, H. Hbner, L. Arnold, R. Krause, and P. Gibbon, "A massively parallel, multi-disciplinary barneshut tree code for extreme-scale n-body simulations," Computer Physics Communications, vol. 183, no. 4, pp. 880 - 889, 2012. [Online]. Available: http://www.sciencedirect.com/science/article/pii/S0010465511004012

[44] M. S. Warren, "2hot: An improved parallel hashed oct-tree nbody algorithm for cosmological simulation," in Proceedings of the International Conference on High Performance Computing, Networking, Storage and Analysis, ser. SC '13. New York, NY, USA: ACM, 2013, pp. 72:1-72:12. [Online]. Available: http://doi.acm.org/10.1145/2503210.2503220

[45] D. Blackston and T. Suel, "Highly portable and efficient implementations of parallel adaptive n-body methods," in Proceedings of the 1997 ACM/IEEE Conference on Supercomputing, ser. SC '97. New York, NY, USA: ACM, 1997, pp. 1-20. [Online]. Available: http://doi.acm.org/10.1145/509593.509597

[46] I. Newton, Philosophiae naturalis principia mathematica. J. Societatis Regiae ac Typis J. Streater, 1687. [Online]. Available: http: //books.google.nl/books?id=-dVKAQAAIAAJ

[47] P. Hut, J. Makino, and S. McMillan, "Building a better leapfrog," ApJL , vol. 443, pp. L93-L96, Apr. 1995.

[48] L. M. Widrow, B. Pym, and J. Dubinski, "Dynamical Blueprints for Galaxies," ApJ , vol. 679, pp. 1239-1259, Jun. 2008.

[49] J. F. Navarro, C. S. Frenk, and S. D. M. White, "The Structure of Cold Dark Matter Halos," ApJ , vol. 462, p. 563, May 1996.

[50] L. Hernquist, "An analytical model for spherical galaxies and bulges," ApJ , vol. 356, pp. 359-364, Jun. 1990.

[51] L. M. Widrow and J. Dubinski, "Equilibrium Disk-Bulge-Halo Models for the Milky Way and Andromeda Galaxies," ApJ, vol. 631, pp. 838-855, Oct. 2005.

[52] J. Makino, "Treecode with a Special-Purpose Processor," Publ. Astr. Soc. Japan, vol. 43, pp. 621-638, Aug. 1991.

[53] T. Antoja, A. Helmi, O. Bienayme, J. Bland-Hawthorn, B. Famaey, K. Freeman, B. K. Gibson, G. Gilmore, E. K. Grebel, I. Minchev, U. Munari, J. Navarro, Q. Parker, W. Reid, G. M. Seabroke, A. Siebert, A. Siviero, M. Steinmetz, M. Williams, R. Wyse, and T. Zwitter, "Kinematic groups beyond the solar neighbourhood with RAVE," MNRAS , vol. 426, pp. L1-L5, Oct. 2012.

[54] W. Dehnen, "The Effect of the Outer Lindblad Resonance of the Galactic Bar on the Local Stellar Velocity Distribution," AJ , vol. 119 pp. 800-812, Feb. 2000.

[55] T. Antoja, F. Figueras, M. Romero-Gómez, B. Pichardo, O. Valenzuela, and E. Moreno, "Understanding the spiral structure of the Milky Way using the local kinematic groups," MNRAS , vol. 418, pp. 1423-1440, Dec. 2011.

[56] S. Portegies Zwart, S. McMillan, S. Harfst, D. Groen, M. Fujii, B. Ó. Nualláin, E. Glebbeek, D. Heggie, J. Lombardi, P. Hut, V. Angelou, S. Banerjee, H. Belkus, T. Fragos, J. Fregeau, E. Gaburov, R. Izzard, M. Jurić, S. Justham, A. Sottoriva, P. Teuben, J. van Bever, O. Yaron, and M. Zemp, "A multiphysics and multiscale software environment for modeling astrophysical systems," New Astronomy, vol. 14, pp. 369-378, May 2009.

[57] S. Portegies Zwart, S. L. W. McMillan, E. van Elteren, I. Pelupessy, and N. de Vries, "Multi-physics simulations using a hierarchical interchangeable software interface," Computer Physics Communications, vol. 183, pp. 456-468, Mar. 2013.
[58] N. Drost, J. Maassen, M. A. J. van Meersbergen, H. E. Bal, F. I. Pelupessy, S. P. Zwart, M. Kliphuis, H. A. Dijkstra, and F. J. Seinstra, "High-performance distributed multi-model / multi-kernel simulations: A case-study in jungle computing," in IPDPS Workshops, 2012, pp. $150-162$. 